\title{
Discussion of "On the Pointlessness of Machine Learning Based Time Delayed Prediction of TBM Operational Data" by Georg H. Erharter and Thomas Marcher
}

\author{
Brian Sheil \\ Royal Academy of Engineering Research Fellow, Department of Engineering Science, University of \\ Oxford, Parks Road, Oxford OX1 3PJ, UK.
}

\section{Introduction}

The recent paper by Erharter and Marcher [1] provides a timely and thought-provoking discussion of the use of machine learning (ML) for time delayed prediction (TDP) of operational data acquired from a tunnel boring machine (TBM). The stated goal of the Erharter and Marcher [1] study is 'to emphasize that ML based TDP for geotechnics is pointless'. To this end, the authors consider the specific problem of forecasting rock mass conditions ahead of the tunnel face using TBM operational data acquired from an Austrian construction site. Three ML based forecasting techniques are evaluated, namely long shortterm memory (LSTM), support vector regression (SVR) and random forests (RF). The authors conclude that while "high accuracy measures can be achieved, there is no predictive value within TDP forecasts' [1]. The purpose of this discussion is to make a case for a more optimistic outlook for the role of $\mathrm{ML}$ forecasting techniques in the tunnelling industry.

\section{ML based forecasting}

$M L$ based forecasting is predicated on the assumption of a sufficient amount of data to describe the modelled system's physics. This approach is therefore only able to capture the 'genuine patterns and relationships which exist in the historical data' [2]. That ML 'predicted sequences are only a slightly different version of the input sequence but shifted into the future' [1] should therefore come as no surprise.

The LSTM, SVR and RF models considered by the authors can be regarded as 'explanatory' models, in the sense that they incorporate underpinning predictor variables into the regression. This contrasts with autoregressive models where the evolving variable of interest is predicted on its own lagged values and no attempt is made to discover underlying causal relationships (e.g. ARIMA; [3]). While explanatory models are useful, particularly in a model interpretability context, a key limitation of these techniques is the need to also forecast the predictor variables as a step to forecasting the variable of interest. Another important assumption is that the system to be forecasted is both well understood and can be well 
described by the adopted predictor variables. However, for tunnelling applications, important predictor variables can be both difficult to quantify and even more difficult to forecast (e.g. TBM driving style). For these reasons, these techniques do not lend themselves easily to long-term forecasting of complex systems such as TBMs.

A further drawback of these techniques is their deterministic basis. These approaches tacitly assume complete confidence of the data acquired from the TBM. In contrast, probabilistic techniques provide a robust approach for treating signal artefacts encountered in noisy construction data and incorporates uncertainty explicitly into the model [4]. One such approach is Gaussian Process (GP) regression which has been successfully applied to forecasting complex systems in a broad range of practical domains including battery health (e.g. Richardson et al. [5]), wind power (e.g. Yan et al. [6]), dam movements (e.g. Lin et al. [7]) and tourism demand (e.g. Wu et al. [8]). GPs provide a method to perform Bayesian inference about functions in a non-parametric way. One of the key aspects of GPs is the use of covariance functions (also known as 'kernels') which encodes prior assumptions about the functions one wishes to learn, in this case the monitored TBM data. The covariance functions can be 'designed' to capture the structure of the monitored data as a proxy for the underlying physics of the system thereby avoiding reliance on algebraic mapping between inputs and outputs. These techniques may prove to be a more robust way forward for longterm forecasting of construction data.

\section{The role of forecasting in tunnelling}

The authors state that the 'goal of forecasting TBM operational data is to identify changing rockmass conditions via changing patterns within the TBM data itself before they are encountered in the excavation itself '[1]. The discusser agrees that the use of TBM operational parameters to forecast geotechnical conditions far ahead of the TBM is indeed likely to be a fruitless endeavour. However, this is not because ML algorithms are incapable of identifying this relationship but because such a relationship simply does not exist. Rather, the combination of $\mathrm{ML}$ forecasting techniques with soil imaging techniques has been shown to be a much more promising avenue of research e.g. Schaeffer \& Mooney [9], Wei et al. [10], Sheil et al. [11].

The discusser also disagrees that geological prediction is the only viable role for forecasting techniques in tunnelling. Accurate forecasting of TBM performance in the short-term is desirable for TBM anomaly detection [12] as well as boulder detection [9]. In this case, the ML model learns 'healthy' behaviour thereby developing a model of 'normality' from which anomalous behaviour may then be inferred. Techniques capable of capturing the structure of 
the data, such as GPs, may also prove useful for long-term forecasting of likely maximum thrust and torque requirements given current 'working' behaviour [4].

\section{References}

[1] G.H. Erharter, T. Marcher, On the pointlessness of machine learning based time delayed prediction of TBM operational data, Autom. Constr. 121 (2021), https://doi.org/10.1016/j.autcon.2020.103443.

[2] R.J. Hyndman, G. Athanasopoulos, Forecasting: principles and practice, (2018), Second edition. OTexts.

[3] G.E. Box, G.M. Jenkins, G.C. Reinsel, Time series analysis: forecasting and control, 734 (2011), John Wiley \& Sons.

[4] B.B. Sheil, Prediction of microtunnelling jacking forces using a probabilistic observational approach. Tunn. Undergr. Space Technology (2020), published online ahead of print. https://doi.org/10.1016/j.tust.2020.103749.

[5] R.R. Richardson, M.A. Osborne, D.A. Howey, Gaussian process regression for forecasting battery state of health, J. Power Sources 357 (2017) 209-219, https://doi.org/10.1016/j.jpowsour.2017.05.004.

[6] J. Yan, K. Li, E.W. Bai, J. Deng, A.M. Foley, A.M., Hybrid probabilistic wind power forecasting using temporally local Gaussian process, IEEE Trans. Sustain. Energy 7 (1) (2015) 87-95, https://doi.org/10.1109/TSTE.2015.2472963.

[7] C. Lin, T. Li, S. Chen, X. Liu, C. Lin, S. Liang, Gaussian process regression-based forecasting model of dam deformation, Neural Comput. Appl., 31 (12) (2019) 85038518, https://doi.org/10.1007/s00521-019-04375-7.

[8] Q. Wu, R. Law, X. Xu, A sparse Gaussian process regression model for tourism demand forecasting in Hong Kong, Expert Syst. Appl. 39 (5) (2012) 4769-4774, https://doi.org/10.1016/j.eswa.2011.09.159.

[9] K. Schaeffer, M.A. Mooney, Examining the Influence of TBM-Ground Interaction on Electrical Resistivity Imaging ahead of the TBM, Tunn. Undergr. Space Technology 58 (2016) 82-98, https://doi.org/10.1016/j.tust.2016.04.003.

[10] L. Wei, D.R. Magee, A.G. Cohn, An anomalous event detection and tracking method for a tunnel look-ahead ground prediction system. Autom. Constr. 91 (2018) 216-225, https://doi.org/10.1016/j.autcon.2018.03.002.

[11] B.B. Sheil, S.K. Suryasentana, M.A. Mooney, H. Zhu, Machine learning to inform tunnelling operations: recent advances and future trends. Proc. ICE - Smart Infrastruct. Constr. (2020), published online ahead of print. https://doi.org/10.1680/jsmic.20.00011. 
[12] B.B. Sheil, S.K. Suryasentana, W-.C. Cheng, An assessment of anomaly detection methods applied to microtunnelling. J. Geotech. Geoenviron. Eng. 146 (9) (2020), https://doi.org/10.1061/(ASCE)GT.1943-5606.0002326. 